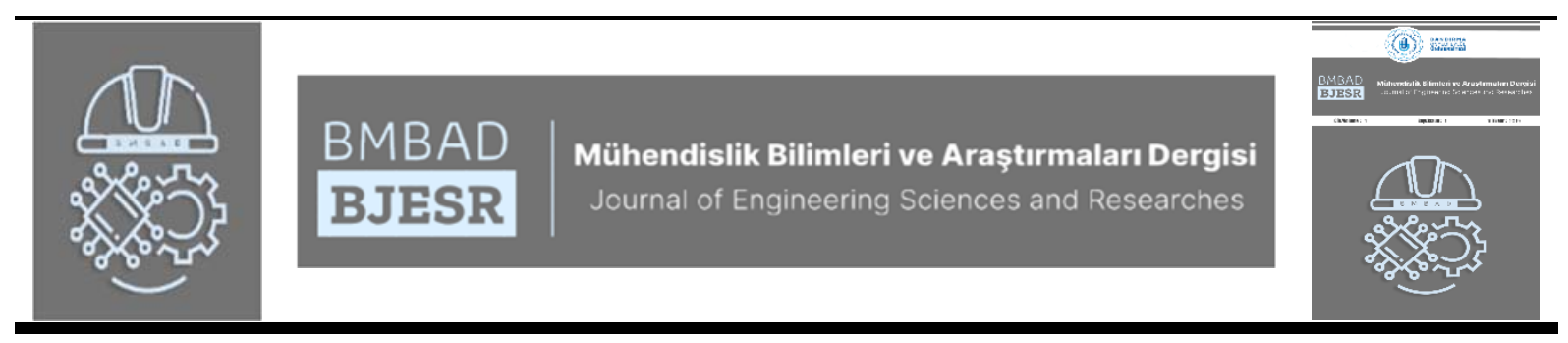

\title{
CMOS Transistörler ile Gerçekleştirilen İkinci Kuşak Akım Taşıyıcısına Ait Performans Parametrelerinin Elman Yapay Sinir Ăg ile İncelenmesi
}

\section{Analysis of Performance Parameter of Second-Generation Current Carrier with CMOS Transistors with Elman Artificial Neural Network}

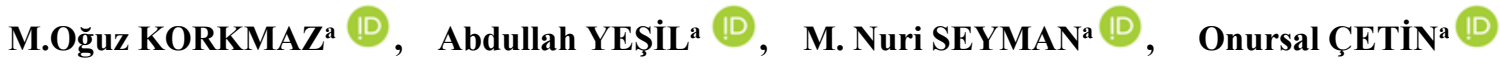 \\ aBandırma Onyedi Eylül Üniversitesi, Mühendislik ve Doğa Bilimleri Fakültesi, Bandırma/Balıkesir, Türkiye
}

mkorkmaz@bandirma.edu.tr, ayesi@bandirma.edu.tr

mseyman@bandirma.edu.tr, ocetin@bandirma.edu.tr

Araştırma Makalesi/Research Article

\begin{tabular}{l}
\hline A R T I C L E I N F O \\
\hline Article history \\
Received : 31 January 2020 \\
Accepted : 10 March 2020 \\
\end{tabular}

Keywords:

Current Conveyor, Artificial Neural Network, Elman, Scaled Conjugate Gradient

\section{A B S T R A C T}

The parasitic impedances cause the system to have varied and/or effected operating characteristics. In order to be able to reduce the effect of parasitic impedances and align the system with ideal operating characteristics, channel width and polarization current of transistors used in circuits can be changed. In this study, in order to be able to reduce the effects of parasitic resistances on second generation current carrying circuit and increase the bandwidth of terminals, an artificial neural network which predicts the appropriate design parameters is created. Created artificial neural network has Elman network structure and involves a single hidden layer having 30 neurons. 12 different training functions are used while training the artificial neural network. The data set used in trainings is created using LTSPICE software. In the data set, there are 60 thousand data having 9 attributes. 54 thousand of these data are used in training while 6 thousand data are reserved for test. The ideal results in the tests were obtained from Scaled Conjugate Gradient training algorithm. Error rate of trained artificial neural network is found to be $4.59 \%$.

(C) 2020 Bandirma Onyedi Eylul University, Faculty of Engineering and Natural Science. Published by Dergi Park. All rights reserved.

\section{Ö Z E T}

Oluşan parazitik empedanslar, sistemin çalışma karakteristiğini değişmesine ve/veya etkilenmesine neden olmaktadır. Parazitik empedansların etkisini azaltabilmek ve sistemi ideal çalışma karakteristiğine yaklaştırabilmek için, devrelerde kullanılan transistörlerin kanal genişlikleri ve kutuplama akımları değiştirilebilir.Yapılan bu çalışmada, ikinci kuşak akım taşıyıcı devresi üzerindeki parazitik dirençlerin etkilerini azaltabilmek ve terminallere ait bant genişliğini arttırmak için, uygun tasarım parametrelerini tahmin eden bir yapay sinir ağı oluşturulmuştur. Oluşturulan yapay sinir ağı, Elman ağ yapısına sahiptir ve 30 nörona sahip tek gizli katman içermektedir. Yapay sinir ağ1 eğitilirken 12 farklı eğitim fonksiyonu kullanılmıştır. Eğitimlerde kullanılan veri seti, LTSPICE programı kullanılarak oluşturulmuştur. Veri setinde 9 adet özniteliğe sahip 60 bin veri bulunmaktadır. Verilerin 54 bini eğitimde kullanılırken 6 bin veri test için ayrılmıştır. Yapılan testlerde en ideal sonucu Scaled Conjugate Gradient eğitim algoritması vermiştir. Eğitilen yapay sinir ağının hata oranı, ortalama \%4,59 olarak bulunmuştur.

C 2020 Bandırma Onyedi Eylül Üniversitesi, Mühendislik ve Dağa Bilimleri Fakültesi. Dergi Park tarafından yayınlanmaktadır. Tüm Hakları Saklıdır. 


\section{GİRIŞ}

Akım modlu devre elemanları; analog sinyal işleme, telekomünikasyon sistemleri gibi elektronik sistemlerde kullanılmaktadır. CMOS teknolojisinin gelişmesiyle birlikte kullanım alanları da artmıştır. Akım modlu devre elemanları, gerilim modlu devre elemanlarına göre daha yüksek frekans bant genişliğine sahip olması, gerilimden bağımsız çalı̧abilmesi, daha iyi lineerlik göstermesi, devre yapısının daha basit olması ve daha büyük yükselme akımına sahip olması gibi özelliklerinden dolayı daha üstün özelliklere sahiptir [1]. Akım modlu devrelerin CCI, CCII, CCIII, DVCC, ICCII, DDCC, DXCCII, FDCCII, CCCII gibi akım taşıyıcı türleri mevcuttur [2]-[10].

Her akım taşıyıcının kendine özgü transfer fonksiyonu olduğu için akım taşıyıcılar ihtiyaca göre seçilmektedir. İdeal devreler için hesaplanan transfer fonksiyonları ile gerçekte kullanılan akım taşıyıcıların transfer fonksiyonları arasında ciddi farklar olabilmektedir. Akım taşıyıcıları etkileyen istenmeyen parazitik empedanslar ve non-ideal kazançlar gibi etmenler yüzünden akım taşıyıcıların çıkış parametreleri değişebilmektedir. Bu durum, akım taşıyıcıların çalıştığı frekans aralıklarının sınırlanmasına ve istenilen şekilde çalışmamasına neden olmaktadır. Akım taşıyıcıların, ideal olmayan koşullarda da çalışabilmesini sağlamak için iç yapısında değişiklikler yapılması gerekmektedir.

Akım taşıyıcıların iç yapısında CMOS transistörler kullanılmaktadır. CMOS transistörlerin kanal boyları, genişliği ve kutuplama akımı parametreleri, ideal tasarım parametrelerine yakın çalışacak şekilde seçilmektedir. Seçimler yapılırken ya transistörlere ait basitleştirilmiş akım gerilim denklemleri kullanılmaktadır ya tasarımcının daha önceki tecrübelerine göre tahmin edilmektedir ya da simülasyon programlarından yardım alınarak optimum tasarım parametreleri bulunmaya çalışılmaktadır.

Yapay sinir ağlarının gelişmesiyle birlikte optimum tasarım parametrelerinin bulunabilmesi için yapay sinir ağları kullanılmaya başlanmıştır. Yapay sinir ağları, beyindeki nöronların yaptığı işlemleri matematiksel olarak taklit ederek, bilgisayarlara kestirim ve genelleme yapabilme yeteneklerini kazandırmayı hedeflemektedir. Yapay sinir ağlarının gelişmesiyle birlikte, veri miktarının çok fazla olduğu haberleşme, biyomedikal gibi alanlarda kullanılmaya başlanmıştır [11]-[15].

İkinci kuşak akım taşıyıcıların performans parametrelerinin tahmin edilebilmesi için yapılan bir çalışmada, ileri beslemeli yapay sinir ağı kullanılmıştır. Yapay sinir ağı, sadece Levenberg-Marquardt eğitim fonksiyonu kullanılarak eğitilmiştir [17].

Yaptığımız bu çalışmada referans [17]'de de kullanılan akım taşıyıcıyla aynı iç yapı kullanılıp, transistor boyutlarının değişim aralığı sıklaştırılarak daha fazla ölçüm alınmışıtır. Ağ yapısı olarak Elman ağı test edilmiştir. Ayrıca yapay sinir ağı 12 farklı eğitim fonksiyonu kullanılarak hem CPU üzerinden hem de GPU üzerinden eğitilmiştir. Eğitilen ağlar kaydedilerek eğitimde hiç kullanılmamış veriler, CMOS içyapısındaki performans parametrelerinin tahmin edilmesinde kullanılmışıır.

\section{YÖNTEM}

Akım taşıyıcılar arasında ikinci kuşak akım taşıyıcı (CCII) yaygın biçimde kullanılmaktadır. CCII, Şekil 1'deki gibi iki girişli ve tek çıkışlı bir yapıya sahiptir.

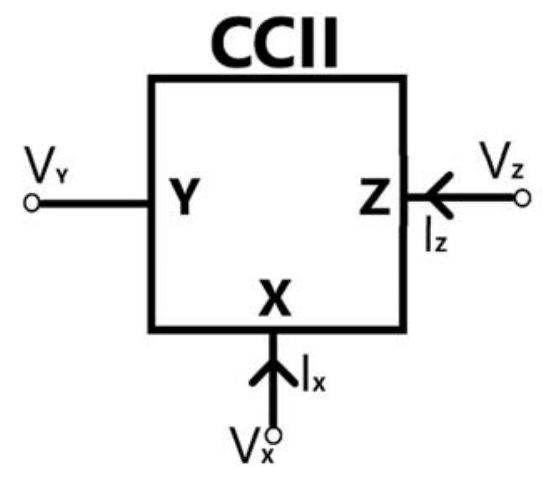

Şekil 1. CCII Sembolü

İdeal bir CCII devresinde $\mathrm{Y}$ terminalinin gerilimi ile $\mathrm{X}$ terminalinin gerilimleri birbirine eşittir. $\mathrm{Y}$ terminalinden akım akmazken, $\mathrm{X}$ terminalinden akan akım ile $\mathrm{Z}$ terminalinden akan akım birbirine eşittir. İdeal olmayan bir CCII devresinin eşdeğer devresi ise Şekil 2'deki gibidir. İdeal olmayan devrenin oluşturulabilmesi için benzetim programında kurulan eşdeğer devreye parazitik dirençler eklenmelidir. Bunun için Şekil 2'de olduğu gibi eşdeğer devrenin girişine seri, çıkışına paralel olacak şekilde parazitik dirençler eklenmektedir. 


\section{CCII}

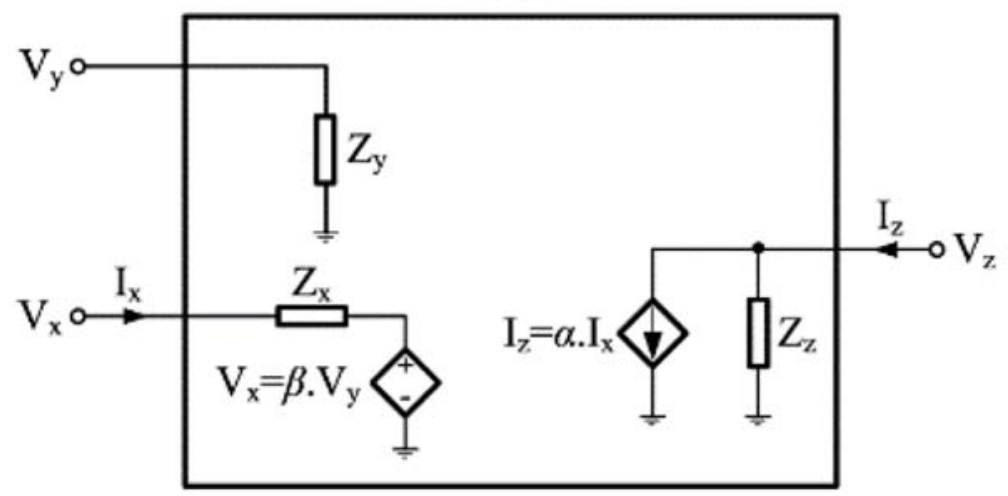

Şekil 2. CCII ideal olmayan eşdeğer devresi [18]

İdeal olmayan eşdeğer devredenin temel denklemleri aşağıdaki gibidir.

$I_{Y}=\frac{V_{Y}}{R_{Y}}$

$V_{X}=\beta V_{Y}+R_{X} I_{X}$

$I_{Z}=\alpha I_{X}+V_{Z} / R_{Z}$

Burada $\alpha$ akım kazancını, $\beta$ ise gerilim kazancını ifade etmektedir. Bu değerler, ideal devrelerde bire eşitken, ideal olmayan devrelerde bire yakın olmaları beklenmektedir.

Bu çalışmada kullanılan veri seti, Şekil 3'teki CCII devresinin iç yapısı kullanarak elde edilmiştir. Veri setinde 5'i giriş ve 4'ü çıkış olmak üzere 9 adet özniteliğe sahip 60 bin veri kullanılmıştır. Verilerin 54 bini eğitim için ayrılırken 6 binini test için ayrılmıştır. Test için ayrılan veriler, eğitimin hiçbir aşamasında kullanılmamıştır. Giriş verisi olarak kullanılan CMOS transistörlerin boyutları belirlenirken aşağıdaki eşitlikler dikkate alınmıştır.

$3 \times \mathrm{M}_{7}=3 \times \mathrm{M}_{10}=\mathrm{M}_{8}=\mathrm{M}_{9}$

$3 \times \mathrm{M}_{16}=3 \times \mathrm{M}_{17}=\mathrm{M}_{2}=\mathrm{M}_{3}$

$3 \times \mathrm{M}_{11}=\mathrm{M}_{12}$

$3 \times \mathrm{M}_{18}=3 \times \mathrm{M}_{19}=\mathrm{M}_{5}=\mathrm{M}_{6}$

Veri setinde giriş verisi olarak; $\mathrm{M}_{5}, \mathrm{M}_{7}, \mathrm{M}_{11}, \mathrm{M}_{16} \mathrm{CMOS}$ transistörlerinin kanal genişlikleri ve kutuplama akımı olan $\mathrm{I}_{\text {ref }}$ akımı kullanılmıştır. Çıkış verisi olarak; X terminaline ait bant genişliğ $\left(\mathrm{f}_{\mathrm{mx}}\right)$, Z terminaline ait bant genişliği $\left(\mathrm{f}_{\mathrm{mz}}\right), X$ terminalindeki seri parazitik direncin büyüklüğü $\left(\mathrm{R}_{\mathrm{x}}\right)$ ve $Z$ terminalindeki paralel parazitik direncin büyüklüğü $\left(\mathrm{R}_{\mathrm{z}}\right)$ kullanılmıştır. Veriler LTSPICE programından elde edilirken denklem (4)-(7) dikkate alınarak, CMOS transistörlerin boyutları $\mathrm{M}_{11}$ transistorun kanal genişliği $5 \mu \mathrm{m}, 10 \mu \mathrm{m}, 20 \mu \mathrm{m}, 30 \mu \mathrm{m}, 40 \mu \mathrm{m}, 50$ $\mu \mathrm{m}$ olarak seçilirken $\mathrm{M}_{5}, \mathrm{M}_{7}$ ve $\mathrm{M}_{16}$ transistörlerinin kanal genişlikleri ise $5 \mu \mathrm{m}$ 'den $50 \mu \mathrm{m}$ 'ye kadar $5 \mu \mathrm{m}$ aralıklarla değiştirilmiştir. Ayrıca, kutuplama akımının değerleri ise $5 \mu \mathrm{A}$ 'den $50 \mu \mathrm{A}$ 'ye kadar $5 \mu \mathrm{A}$ aralıklarla değiştirilmiştir.

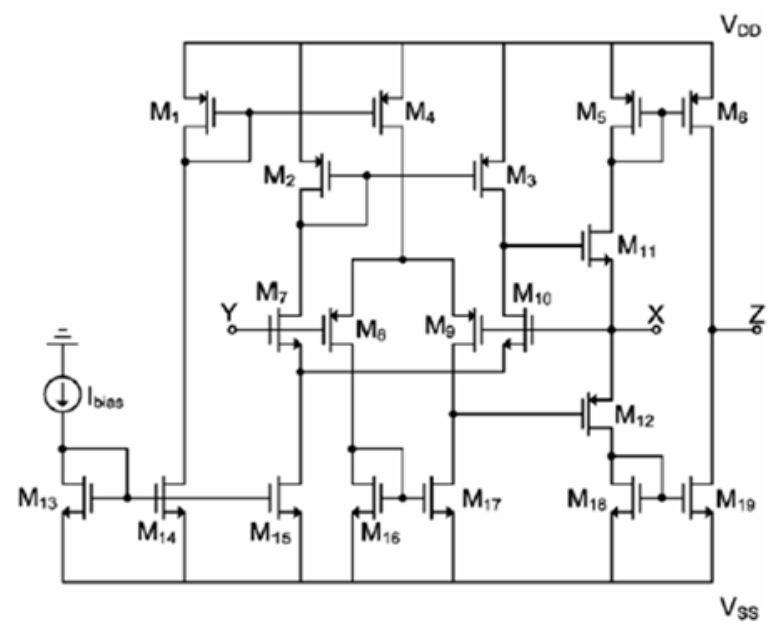

Şekil 3. CCII akım taşıyıcının iç yapısı [19] 
Performans parametrelerini belirlemek istediğimiz akım taşıyıcının verilerini kullanarak, Elman ağ yapısına sahip bir yapay sinir ağı eğitilmiştir. Yapay sinir ağında yapılan tahminlerin yüksek doğruluk oranına sahip olması ve hesaplama sürelerinin düşük olabilmesi için en uygun eğitim fonksiyonunun ve transfer fonksiyonunun belirlenmesi gerekmektedir.

Bu çalışmada eğitim fonksiyonu olarak Regularization, Polak-Ribiére Conjugate Gradient, BFGS Quasi-Newton, Conjugate Gradient with Powell/Beale Restarts, Levenberg-Marquardt, Bayesian Fletcher-Powell Conjugate Gradient, One Step Secant, Variable Learning Rate Gradient Descent, Gradient Descent with Momentum, Resilient Backpropagation, Scaled Conjugate Gradient ve Gradient Descent fonksiyonları test edilmiştir.

Transfer fonksiyonu olarak, Hard-Limit transfer fonksiyonu, Log-Sigmoid transfer fonksiyonu, Linear transfer fonksiyonu, Radial Basis transfer fonksiyonu ve Hyperbolic Tangent Sigmoid transfer fonksiyonu test edilmiştir. Yapılan testlerde Hyperbolic Tangent Sigmoid transfer fonksiyonun diğer transfer fonksiyonlarına göre daha başarılı olduğu görüldüğünden yapay sinir ağının bütün katmanlarında kullanılmıştır.

\section{BENZETIMM ÇALIŞMALARI}

Bu çalışmada Şekil 4'te verilen Elman ağı, MATLAB [Lisans No: 40692431] ortamında 12 farklı eğitim fonksiyonuyla test edilmiştir. Elman ağının tüm katmanlarında transfer fonksiyonu olarak Hyperbolic Tangent Sigmoid transfer fonksiyonu kullanılmıştır. Ağ oluşturulurken 30 nöronlu tek katman kullanılmıştır. Elman ağı, ileri beslemeli ve geri beslemeli bağlantılara sahiptir. Eğitim sırasında ileri beslemeli bağlantılar ve standart geri yayılım algoritması kullanmaktadır. Geri beslemeli bağlantılar ise sabit kalmaktadır.

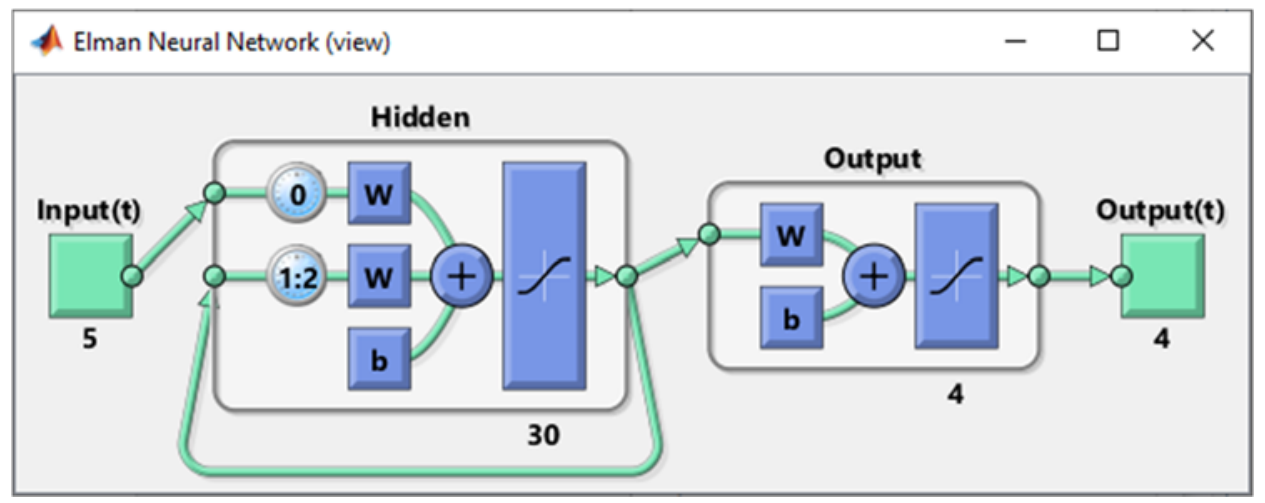

Şekil 4. Elman ağ yapısı

Elman yapay sinir ağı ve akım taşıyıcıya ait veri setleri ekran kartı üzerinden hesaplanarak elde edilen sonuçlar Tablo 1'de verilirken, işlemci üzerinden yapılan hesaplamaların sonuçları Tablo 2'de verilmiştir. Tablolarda, 12 farklı eğitim fonksiyonunun sonuçları bulunmaktadır. Min-Max yüzdelik hata oranı sütununda, eğitim algoritmalarının testleri sırasında karşılaşılan en küçük yüzdelik hata oranı ile en büyük yüzdelik hata oranı belirtilmiştir. Eğitim sayısı, eğitim fonksiyonunun kaç iterasyon sonunda doğrulama kontrol koşulunu sağlandığını göstermektedir. Eğitim süresi, doğrulama kontrol koşullarının ne kadar sürede sağlandığını göstermektedir. Ortalama hata oranı ise, eğitime sokulmayan 6 bin verinin, eğitimi tamamlanmış yapay sinir ağı üzerinde hesaplanmasıyla elde edilen hata oranlarının aritmetik ortalamasıdır.

Tablo 1. GPU ile hesaplanmış Elman ağ yapısı

\begin{tabular}{lllll}
\hline Eğitim Fonksiyonu & $\begin{array}{l}\text { Min-Max Yüzdelik } \\
\text { Hata Oranı }\end{array}$ & $\begin{array}{l}\text { Eğitim } \\
\text { Sayısı }\end{array}$ & $\begin{array}{l}\text { Eğitim } \\
\text { Süresi }\end{array}$ & $\begin{array}{l}\text { Ortalama Hata } \\
\text { Oranı }\end{array}$ \\
\hline Levenberg-Marquardt & Hesaplanamamaktadır & - & - & - \\
\hline Bayesian Regularization & Hesaplanamamaktadır & - & - & - \\
\hline BFGS Quasi-Newton & Hesaplanamamaktadır & - & - & - \\
\hline Resilient Backpropagation & $\% 1,06-\% 54,46$ & 2000 & $00: 00: 50$ & $\% 10,47$ \\
\hline Scaled Conjugate Gradient & $\% 0,37-\% 31,45$ & 2000 & $00: 02: 56$ & $\% 4,59$ \\
\hline $\begin{array}{l}\text { Conjugate Gradient with } \\
\text { Powell/Beale Restarts }\end{array}$ & $\% 4,97-\% 324,08$ & 59 & $00: 00: 06$ & $\% 50,57$
\end{tabular}




\begin{tabular}{|c|c|c|c|c|}
\hline $\begin{array}{l}\text { Fletcher-Powell Conjugate } \\
\text { Gradient }\end{array}$ & $\% 0,71-\% 216,54$ & 2000 & 00:02:49 & $\% 9,63$ \\
\hline $\begin{array}{l}\text { Polak-Ribiére Conjugate } \\
\text { Gradient }\end{array}$ & $\% 0,63-\% 46,56$ & 2000 & $00: 02: 54$ & $\% 6,94$ \\
\hline One Step Secant & $\% 0,63-\% 100,17$ & 2000 & $00: 02: 53$ & $\% 8,97$ \\
\hline $\begin{array}{l}\text { Variable Learning Rate } \\
\text { Gradient Descent }\end{array}$ & $\% 0,84-\% 225,49$ & 2000 & $00: 00: 51$ & $\% 17,89$ \\
\hline $\begin{array}{l}\text { Gradient Descent with } \\
\text { Momentum }\end{array}$ & $\% 2,64-\% 1115,36$ & 2000 & 00:00:52 & $\% 53,30$ \\
\hline Gradient Descent & $\% 2,22-\% 980,52$ & 2000 & 00:00:52 & $\% 55,03$ \\
\hline
\end{tabular}

Tablo 2. CPU ile hesaplanmış Elman ağ yapısı

\begin{tabular}{|c|c|c|c|c|}
\hline Eğitim Fonksiyonu & $\begin{array}{l}\text { Min-Max Yüzdelik } \\
\text { Hata Oranı }\end{array}$ & $\begin{array}{l}\text { Ĕgitim } \\
\text { Sayısı }\end{array}$ & $\begin{array}{l}\text { Eğitim } \\
\text { Süresi }\end{array}$ & $\begin{array}{l}\text { Ortalama Hata } \\
\text { Oranı }\end{array}$ \\
\hline Levenberg-Marquardt & $\% 0,39-\% 44,93$ & 468 & 08:01:41 & $\% 5,75$ \\
\hline Bayesian Regularization & $\% 0,50-\% 44,51$ & 418 & $00: 18: 35$ & $\% 9,52$ \\
\hline BFGS Quasi-Newton & $\% 4,60-\% 2676,92$ & 2000 & 02:08:22 & $\% 135,05$ \\
\hline Resilient Backpropagation & $\% 0,85-\% 54,22$ & 2000 & 00:02:04 & $\% 9,47$ \\
\hline Scaled Conjugate Gradient & $\% 0,59-\% 44,99$ & 2000 & $00: 03: 54$ & $\% 6,19$ \\
\hline $\begin{array}{l}\text { Conjugate Gradient with } \\
\text { Powell/Beale Restarts }\end{array}$ & $\% 0,91-\% 52,38$ & 2000 & $00: 06: 55$ & $\% 6,87$ \\
\hline $\begin{array}{l}\text { Fletcher-Powell Conjugate } \\
\text { Gradient }\end{array}$ & $\% 5,87-\% 3117,44$ & 2000 & $00: 05: 54$ & $\% 145,66$ \\
\hline $\begin{array}{l}\text { Polak-Ribiére Conjugate } \\
\text { Gradient }\end{array}$ & $\% 5,46-\% 2728,39$ & 210 & 00:00:49 & $\% 161,97$ \\
\hline One Step Secant & $\% 0,46-\% 132,28$ & 2000 & 00:06:47 & $\% 9,59$ \\
\hline $\begin{array}{l}\text { Variable Learning Rate } \\
\text { Gradient Descent }\end{array}$ & $\% 0,70-\% 217,65$ & 2000 & $00: 01: 55$ & $\% 15,46$ \\
\hline $\begin{array}{l}\text { Gradient Descent with } \\
\text { Momentum }\end{array}$ & $\% 3,28-\% 698,37$ & 2000 & $00: 01: 56$ & $\% 46,66$ \\
\hline Gradient Descent & $\% 13,65-\% 1609,27$ & 2000 & 00:01:56 & $\% 90,37$ \\
\hline
\end{tabular}

\section{VERILERIIN DEĞERLENDİRILMESİ VE BULGULAR}

Benzetim çalışmalarındaki hesap işlemleri hem ekran kartı üzerinden hem de işlemci üzerinden yapılıp incelenmiştir. Yapılan çalışmalarda Levenberg-Marquardt, Bayesian Regularization ve BFGS Quasi-Newton eğitim algoritmalarının ekran kartı üzerinden hesaplanamadığı için bu 3 eğitim fonksiyonu sadece işlemci üzerinden hesaplanmıştır.

Ekran kartı üzerinden yapılan hesaplamaların, işlemci üzerinden yapılan hesaplamalara göre genellikle çok daha hızlı olduğu görülmüştür. Ekran kartları üzerinden oluşturulan yapay sinir ağları ile işlemciler üzerinden oluşturulan yapay sinir ağları arasındaki hata oranları birbirine oldukça yakındır. $\mathrm{Bu}$ farkın temel nedeni, donanımların değişmesinden ziyade eğitim için ayrılan verilerin farklı sıralarla eğitime sokulmasıdır. Aynı benzetim çalışması art arda yapıldığında dahi sonuçlar arasında yaklaşık \%1 oranında farklılıklar meydana gelebilmektedir. 
Elman Ağ yapısı ile yapılan benzetim çalışmaları incelendiğinde eğitim fonksiyonlarının ortalama hata oranları \%4,59 ile \%162,0 arasında gerçekleşmiştir. En düşük ortalama hata oranı \%4,59 ile Scaled Conjugate Gradient eğitim fonksiyonuyla eğitilen yapay sinir ağında gerçekleşmiştir. Eğitim, 2000 iterasyonda tamamlanmıştır ve eğitim 2 dakika 56 saniye sürmüştür. Eğitilen yapay sinir ağının hata histogramı Şekil 5'de gösterilmiştir. Histograma göre hata oranı \%1,5 ile \%4,5 arasında yoğunlaşmıştır.

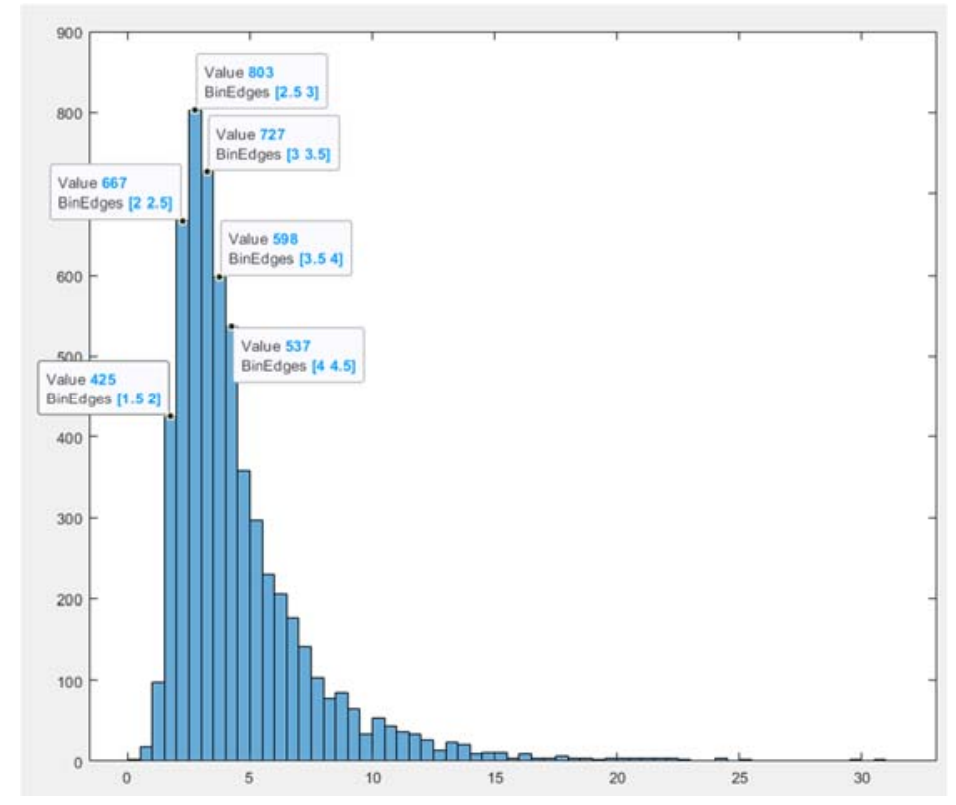

Şekil 5. Elman ağ yapısında Scaled Conjugate Gradient eğitim algoritmasının hata histogramı

Scaled Conjugate Gradient algoritması ile eğitilmiş Elman ağ yapısındaki bir yapay sinir ağı ile örnek bir hesaplama yapılmıştır. Elde edilen sonuçlar Tablo 3'deki gibidir.

Tablo 3. Scaled Conjugate Gradient eğitim algoritması ile hesaplanan veriler

\begin{tabular}{llllllllll}
\hline & \multicolumn{4}{c}{ Giriş Verileri } & \multicolumn{4}{c}{ Çıkış Verileri } \\
\cline { 2 - 10 } & $\mathrm{M}_{11}$ & $\mathrm{M}_{7}$ & $\mathrm{M}_{16}$ & $\mathrm{M}_{18}$ & $\mathrm{I}_{\text {bias }}$ & $\mathrm{f}_{\mathrm{mx}}$ & $\mathrm{f}_{\mathrm{mz}}$ & $\mathrm{R}_{\mathrm{X}}$ & $\mathrm{R}_{\mathrm{Z}}$ \\
\hline Birimler & {$[\mu \mathrm{m}]$} & {$[\mu \mathrm{m}]$} & {$[\mu \mathrm{m}]$} & {$[\mu \mathrm{m}]$} & {$[\mu \mathrm{A}]$} & {$[\mathrm{MHz}]$} & {$[\mathrm{MHz}]$} & {$[\Omega]$} & {$[\mathrm{k} \Omega]$} \\
\hline Gerçek Değerler & 40 & 10 & 45 & 35 & 25 & 332,267 & 555,621 & 3,09244 & 25,84240 \\
\hline Hesaplanan Değerler & 40 & 10 & 45 & 35 & 25 & 331,591 & 522,844 & 3,09824 & 28,43718 \\
\hline Yüzdelik Fark & - & - & - & - & - & $\% 0,2$ & $\% 5,9$ & $\% 0,19$ & $\% 10,04$ \\
\hline
\end{tabular}

Tablo 3'de yapılan örnek hesaplamada, gerçek değerler ile hesaplanan değerler arasındaki ortalama yüzdelik fark $\%$ 4,08 olarak hesaplanmıştır.

\section{SONUÇ}

Yapılan bu çalışmada, ikinci kuşak akım taşıyıcıların performans parametrelerinin belirlenebilmesi için yapay sinir ağlarından faydalanılmıştır. Yapay sinir ağı olarak Elman ağ modeli kullanılmıştır. Ağ modeli de 30 nörona sahip tek gizli katmandan oluşmaktadır. A ̆g eğitilirken 12 farklı eğitim fonksiyonu kullanılarak en ideal eğitim fonksiyonunun tespit edilmesi amaçlanmıştır. Ayrıca CPU ve GPU için ayrı ayrı hesaplamalar yapılmıştır. Ağlarda bulunan katmanların tamamında transfer fonksiyonu olarak Hyperbolic Tangent Sigmoid transfer fonksiyonu kullanılmıştır. Eğitilen ağlar test edildiğinde hata oranının yaklaşık \%2 civarında olduğu görülmüştür.

$\mathrm{Bu}$ çalışmada, yapay sinir ağı modellerinden sadece Elman ağı analiz edilmiştir. Feedforward, Design Generalized Regression, Function Fitting, Radial Basis, Learning Vector Quantization, Self Organizing Map, Jordan gibi diğer yapay sinir ağ modelleri de analiz edilerek daha iyi sonuçlar elde edilebilir.

\section{Teșekkür}

Bu çalışma Bandırma Onyedi Eylül Üniversitesi Bilimsel Araştırma Projeleri Koordinasyon Birimi tarafından desteklenmiştir. Proje Numarası: BAP-18-DF-1003-004. 


\section{KAYNAKÇA}

[1] A. A. El-Adawy, A. M. Soliman, ve H. O. Elwan, “A novel fully differential current conveyor and applications for analog VLSI," IEEE Trans. Circuits Syst. II Analog Digit. Signal Process., vol. 47, no. 4, pp. 306-313, 2000.

[2] W. Chiu, S.-I. Liu, H.-W. Tsao, ve J.-J. Chen, "CMOS differential difference current conveyors ve their applications," IEE Proc. - Circuits, Devices Syst., vol. 143, no. 2, pp. 91-96, 1996.

[3] F. Kacar, B. Metin, ve H. Kuntman, "A new CMOS dual-X second generation current conveyor (DXCCII) with an FDNR circuit application," AEU Int. J. Electron. Commun., vol. 64, no. 8, pp. 774778, 2010.

[4] A. Fabre, O. Saaid, F. Wiest, ve C. Boucheron, "High frequency applications based on a new current controlled conveyor," IEEE Trans. Circuits Syst. I Fundam. Theory Appl., vol. 43, no. 2, pp. 82-91, 1996.

[5] H. A. Alzaher, H. Elwan, ve M. Ismail, “A cmos fully balanced second-generation current conveyor," IEEE Trans. Circuits Syst. II Analog Digit. Signal Process., vol. 50, no. 6, pp. 278-287, 2003.

[6] S. Awad Mahmoud, "New fully-differential cmos second-generation current conveyer," ETRI J., vol. 28, no. 4, pp. 495-501, 2006.

[7] E. Arslan, B. Metin, N. Herencsar, J. Koton, A. Morgul, ve O. Cicekoglu, "High performance wideband CMOS CCI and its application in inductance simulator design," Adv. Electr. Comput. Eng., vol. 12, no. 3, pp. 21-26, 2012.

[8] S. Minaei, "A new high performance CMOS third generation current conveyor (CCIII) and its application," Electr. Eng. (Archiv fur Elektrotechnik), vol. 85, no. 3, pp. 147-153, 2003.

[9] M. A. Ibrahim, H. Kuntman, S. Ozcan, O. Suvak, ve O. Cicekoglu, "New first-order inverting-type second-generation current conveyor-based all-pass sections including canonical forms," Electr. Eng., vol. 86, no. 5, 2004.
[10] S. Minaei ve C. Temizyurek, "Dual input all-pass filter using DVCC," in SCS 2003. International Symposium on Signals, Circuits and Systems. Proceedings, vol. 2, pp. 477-480, 2003.

[11] O. Çetin, F. Temurtaş, ve Ş. Gülgönül, “An application of multilayer neural network on hepatitis disease diagnosis using approximations of sigmoid activation function," Dicle Med. J. / Dicle Tip Derg., vol. 42, no. 2, pp. 150-157, 2015.

[12] M. N. Seyman ve N. Taspinar, "Radial Basis Function Neural Networks for Channel Estimation in MIMO-OFDM Systems," Arab. J. Sci. Eng., vol. 38, no. 8, pp. 2173-2178, 2013.

[13] A. Dalcalı, O. Çetin ve F. Temurtaş, "Investigation of current-force relationship in an electromagnetic launcher," Electronic Letters on Science and Engineering, vol. 15, no. 1, pp. 21-27, 2019.

[14] F. Temurtas, "A comparative study on thyroid disease diagnosis using neural networks," Expert Syst. Appl., vol. 36, no. 1, pp. 944-949, 2009.

[15] H. Makas ve N. Yumusak, "Classification using neural networks trained by swarm intelligence," Electronic Letters on Science and Engineering, vol. 13 , no. 1, pp. 16-30, 2007

[16] G. Ren, Y. Cao, S. Wen, T. Huang, ve Z. Zeng, “A modified elman neural network with a new learning rate scheme," Neurocomputing, vol. 286, pp. 11-18, 2018.

[17] A. Yeşil, Y. Çelik, ve F. Temurtaş, "Yapay sinir ağları yöntemi ile ikinci kuşak akım taşıyıcının performans parametrelerinin tahmin edilmesi," Mühendislik Bilimleri ve Araştırmaları Dergisi, vol. 1, no. 1, pp. 13-23, 2019.

[18] E. Arslan ve A. Morgül, "Self-biasing current conveyor for high frequency applications," J. Circuits, Syst. Comput., vol. 21, no. 05, 2012.

[19] E. Arslan ve A. Morgül, "Wideband current conveyor with rail to rail input stage," ELECO, 5th Int. Conf. Electr. Electron. Eng., pp. 66-70, 2007. 